\title{
Studies on the Formation of Regular Stacks of Benzyl Groups in Racemic Poly( $\gamma$-benzyl glutamate) Films
}

\author{
Junji Watanabe, Kazumichi IMAI, Kenichi KosaKa,* \\ Akihiro ABE, and Ichitaro UEMATSU \\ Department of Polymer Chemistry, Tokyo Insitute of Technology, \\ Ookayama, Merugo-ku, Tokyo 152, Japan. \\ * Japan Electron Optics Laboratory, \\ 1418 Nakagami-cho, Akishima, Tokyo 196, Japan.
}

(Received November 29, 1980)

KEY WORDS ${ }^{13} \mathrm{C}$ Nuclear Magnetic Resonance / Excimer Emission /
Benzyl Group / Racemic Mixture / Poly $(\gamma$-benzyl glutamate $)$

The poly( $\gamma$-benzyl glutamate) (PBG) film comprising a racemic mixture of $\mathrm{L}$ - and $\mathrm{D}$-isomers has been known to exhibit physical properties quite different from those of poly( $\gamma$-benzyl L-glutamate) (PBLG). A simple but important structural characteristic of the racemic form may be found in the $\mathrm{X}$ ray diffraction diagram; i.e., the $10.5 \AA$ meridional reflection appearing in this diagram cannot be explained simply in terms of the $\alpha$-helical backbone conformation of the PBLG molecule. ${ }^{1,2}$ Mitsui et $a l .{ }^{1}$ studied a PBG fiber comprising a racemic mixture of $\mathrm{L}$ - and D-isomers, and concluded that the intermolecular side-chain interaction between these two isomers allows benzyl groups to be so arranged as to give the observed "extra" layer lines. Later, Squire et al..$^{2}$ proposed a structural model in which the benzyl groups of the side chain sticking out from two $\alpha$-helical molecules of the opposite sense situated next to each other are piled up in an alternative fashion to form a regular stack of aromatic rings. Such a stack is regarded as the origin of the aforementioned "extra" reflection observed in the X-ray diagram.

Uematsu et al. ${ }^{3}$ found that this meridional reflection disappears at $97^{\circ} \mathrm{C}$ on heating and reappears at $73^{\circ} \mathrm{C}$ on cooling. From $\mathrm{DSC}^{3}$ and dilatometric ${ }^{4}$ measurements, they concluded that such a phenomenon should be related to the first order transition arising from the cooperative transformation of the regular stacks of aromatic rings. From viscoelastic and dielectric measurements,
Uematsu et al. ${ }^{3}$ and Hikichi et al. ${ }^{5}$ reported that the strength of the relaxation associated with the sidechain rotation is smaller for racemic PBG than for enantiomorphic PBLG at room temperature. On heating the sample, such a restriction imposed on the side-chain rotation seems to be removed at about the above-mentioned transition temperature. A sharp drop in Young's modulus and an abrupt increase in the dielectric constant take place simultaneously. All these observations strongly suggest that the regular stacks of aromatic rings occur between the two enantiomers: one with the righthanded (L-isomer) and the other with the lefthanded $\alpha$-helical backbone (D-isomer).

If this model is realistic, the benzyl groups involved in the regular stacks should be less mobile than those free from such a restriction. Under these conditions, we may expect the formation of an excimer between adjacent aromatic rings fixed in the stacked configuration. Upon "melting," these stacked benzyl groups may acquire freedom of rotating independent of each other. In this study, we employed ${ }^{13} \mathrm{C}$-NMR to monitor the mobility of the benzyl groups as a function of temperature. Studies were also made for the fluorescence excimer emission characteristic of the racemic mixture of PBG.

\section{EXPERIMENTAL}

The PBLG and poly( $\gamma$-benzyl D-glutamate) (PBDG) samples used in this study were prepared 
by polymerization of the corresponding $N$ carboxyanhydrides in dioxane, triethylamine being used as the initiator. The molecular weights determined from viscosity measurements in dichloroacetic acid were 300,000 and 270,000 for PBLG and PBDG, respectively. A racemic mixture was prepared by mixing equal amounts (by weight) of PBLG and PBDG. Films were cast from chloroform solutions. The films obtained from this racemic mixture were found to exhibit structural characteristics and physical properties noted above. All the samples were annealed at $150^{\circ} \mathrm{C}$ for $1 \mathrm{~h}$ in vacuo prior to measurements.

High-resolution ${ }^{13} \mathrm{C}$ FT NMR measurements were carried out at $25 \mathrm{MHz}$ by a JEOL FX-100 spectrometer equipped with a Fourier transform system. NMR spectra were obtained for films held in a $10 \mathrm{~mm}$ sample tube over the temperature range from -40 to $130 \mathrm{C}$.

UV absorption spectra were measured by a Hitachi 556 double wavelength spectrophotometer. Fluorescence emission and excitation spectra were observed by a Shimadzu spectrofluorophotometer RF-502 equipped with a compensator. In these measurements, films were held in a quartz tube sealed under nitrogen atmosphere. A quartz cell with a $1-\mathrm{cm}$ path was used for the solutions. Chloroform (Tokyo Kasei, spectroscopic grade) was used as the solvent without further purification.

\section{RESULTS AND DISCUSSION}

\section{${ }^{13} C-N M R$ Spectra}

An example of the ${ }^{13} \mathrm{C}-\mathrm{NMR}$ spectra for a PBLG film is shown in Figure 1. The peak with the highest intensity (indicated by the arrow) is assigned to the phenyl carbons. The logarithm of the line width $\left(\log \Delta H_{1 / 2}\right)$ of this peak varies with temperature as shown in Figure 2, along with the results for a racemic (PBLG + PBDG) sample. The curves thus derived for these two samples are distinguishable from each other only in the temperature region from 0 to $100^{\circ} \mathrm{C}$, where the line width $\Delta H_{1 / 2}$ decreases gradually, but in a considerable amount, with increase in temperature.

From the analysis of the broad line ${ }^{1} \mathrm{H}-\mathrm{NMR}$ spectra for PBLG, Kail et al. ${ }^{6}$ and Hikichi et al ${ }^{7,8}$ concluded that the large decrease in the second moment observed over the range from -20 to $100 \mathrm{C}$ is caused by the onset of the internal rotation

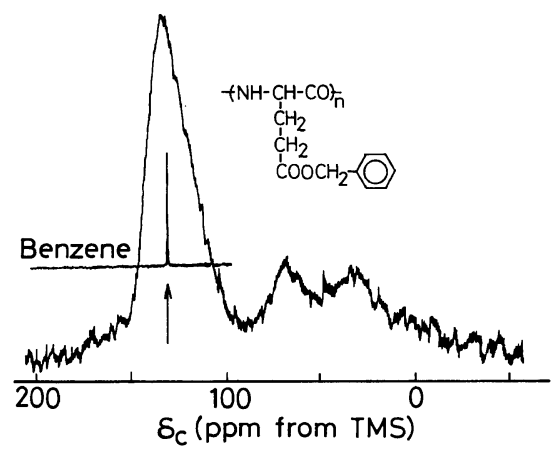

Figure 1. ${ }^{13} \mathrm{C}-\mathrm{NMR}$ spectrum at $100^{\circ} \mathrm{C}$ for PBLG film. Also shown for comparison is the spectrum observed for benzene as indicated by the arrow. The largest peak in the PBLG spectrum may thus be identified as that due to aromatic (phenyl) carbons.

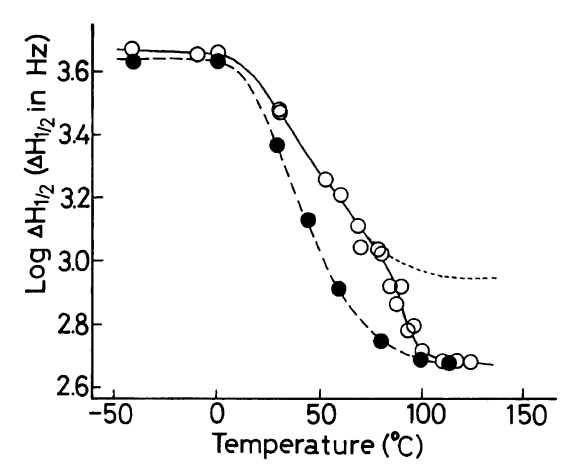

Figure 2. Temperature dependence of the line width $\left(\log \Delta H_{1 / 2}\right)$ for the phenyl peak: - - racemic form (PBLG + PBDG); ----, enantiomorphic form (PBLG). The dotted curve was drawn as noted in the text.

of the entire side chain. Relaxation arising from this source may be responsible for the narrowing of the line width for PBLG as shown by the broken curve in Figure 2. The observed delay in the motional narrowing for the racemic mixture, as measured by the difference between the two curves given in Figure 2 , reaches a maximum at around $70^{\circ} \mathrm{C}$. This delay may be caused by the regular stacks of benzyl groups formed sporadically in the racemic sample. ${ }^{2}$ It is then quite reasonable to assume that a sharp decrease in the solid curve in Figure 2, which becomes marked above $70^{\circ} \mathrm{C}$, corresponds to the "melting" of these regular stacks of benzyl groups. The dotted curve in Figure 2 was drawn by extending the upper portion of the solid curve, where the "melting" is yet ineffective. The slope of the dotted 


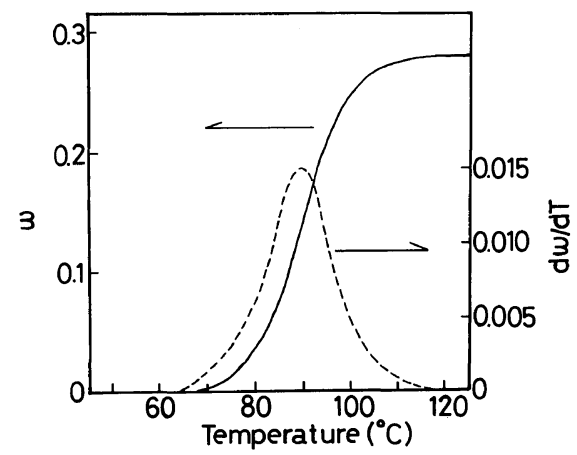

Figure 3. The "melting" curve (shown by the solid curve) for the transition of the regular stacks of benzyl groups as estimated by taking the difference $(\omega)$ between the solid and dotted curves of Figure 2. The broken curve was derived by the differentiation $(\mathrm{d} \omega / \mathrm{d} T)$ of the solid curve.

curve was determined somewhat arbitrarily: in practice, the general trend of the broken curve (PBLG) was followed assuming that this curve represents the characteristic behavior of the side chain which is free from the stacking of benzyl groups. The dotted curve derived in this manner indicates a hypothetical locus, along which the line width $\left(\log \Delta H_{1 / 2}\right)$ of the racemic PBG sample should vary with temperature if the "melting" point of the stacks of benzyl groups was much higher $\left(\gg 100^{\circ} \mathrm{C}\right)$. The effect arising from the melting of the stacks of benzyl groups may thus be estimated by taking the difference (denoted by $\omega$ ) between the dotted and solid curves. This difference is shown by the solid curve in Figure 3. Differentiation $(\mathrm{d} \omega / \mathrm{d} T)$ yields a curve having a peak at $90^{\circ} \mathrm{C}$ as indicated by the broken line. When combined with the results of DSC measurements, which exhibit an endothermic transition peak at about $95^{\circ} \mathrm{C}$ on heating, we come to the conclusion that the liberation of benzyl groups from the stacked configuration proceeds in a cooperative fashion. The "melting" curves as estimated above (in Figure 3) are qualitatively consistent with those observed by using DSC. ${ }^{3}$

\section{Fluorescence Spectra}

Some typical examples of the absorption and fluorescence spectra are shown in Figures 4 and 5, respectively. In the dilute PBLG solution in chloroform $\left(3 \times 10^{-3} \mathrm{M}\right)$, an absorption band due to the benzyl chromophore was observed at around

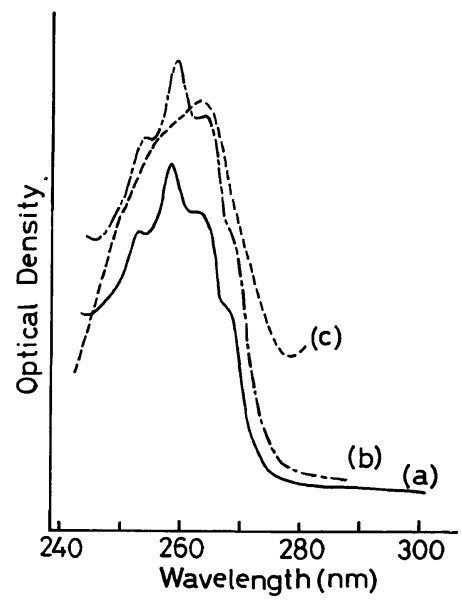

Figure 4. Absorption spectra for (a) PBLG solution in chloroform $\left(3 \times 10^{-3} \mathrm{M}\right)$ and (b) racemic PBG film. Curve (c) represents the excitation spectrum measured for a film of racemic PBG with the monitoring wavelength of $330 \mathrm{~nm}$.

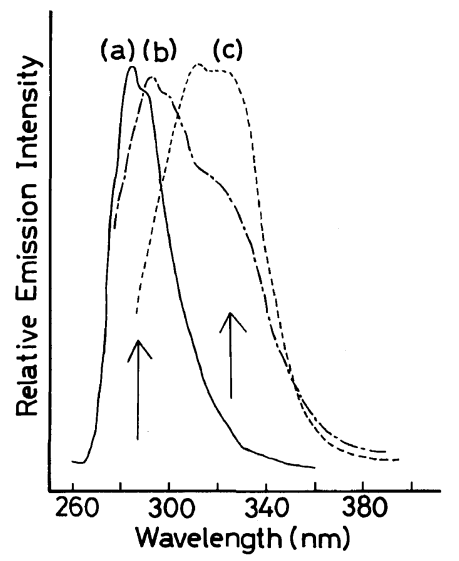

Figure 5. Fluorescence spectra for (a) PBLG solution in chloroform $\left(3 \times 10^{-3} \mathrm{M}\right)$, (b) PBLG film, and (c) racemic $P B G$ film (excitation at $260 \mathrm{~nm}$ ). The arrows positioned at around $285 \mathrm{~nm}$ and around $330 \mathrm{~nm}$ show the normal and excimer emissions, respectively.

$260 \mathrm{~nm}$ (curve (a) in Figure 4), and a somewhat structured fluorescence band at about $285 \mathrm{~nm}$ (curve (a) in Figure 5). ${ }^{9}$ The shape of the fluorescence band was found to alter little with concentration in the range from $5 \times 10^{-4}$ to $5 \times 10^{-3} \mathrm{M}$. This fluorescence band, which is a simple mirror image of the absorption band, may be regarded as the normal emission.

In the case of PBLG film, an additional emission 

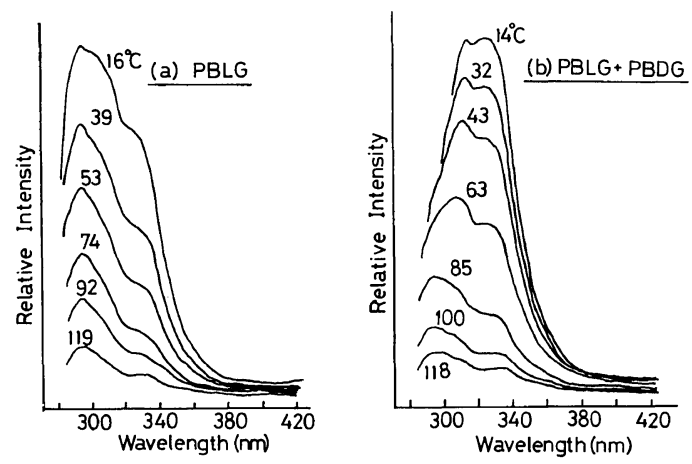

Figure 6. Variation of the fluorescence spectrum with temperature: (a) PBLG film and (b) racemic PBG film.

is noticeable at around $330 \mathrm{~nm}$ as a shoulder on the normal emission band (curve (b) in Figure 5). The intensity of this emission increases markedly in the spectrum (curve (c) in Figure 5) obtained for the film comprising an equimolar mixture of PBLG and PBDG. An excitation spectrum taken with the monitoring wavelength of $330 \mathrm{~nm}$ for the racemic film compares favorably with the corresponding absorption spectrum as can be seen in Figure 4 . These observations suggest that the fluorescence emission at $330 \mathrm{~nm}$ is due to the excimer formed by the interaction between benzyl groups kept within a short distance in the hexagonal arrangement of polypeptide molecules. ${ }^{1,2}$ This is quite consistent with the structural model for the racemic mixture which was thoroughly discussed in the preceding section: regular stacks of benzyl groups ${ }^{2}$ with an interplanar separation of $3-4 \AA$ should enhance formation of the excimer. However, the existence of such stacks of benzyl groups may not be the prerequisite for the excimer formation. A weak emission observed at about $330 \mathrm{~nm}$ in the PBLG film is probably caused by the excimer formed eventually in the two-dimensional hexagonal array of the enantiomorphic molecules. The fact that only the normal emission was observed for the dilute solution of PBLG suggests that the spatial arrangement of benzyl groups prescribed by the $\alpha$-helical main-chain conformation is not adequate for the intramolecular excimer formation. Thus, the excimers formed in the solid state should be derived almost exclusively from the intermolecular interaction.

As the temperature increases, the intensity of the excimer emission of racemic PBG tends to decrease as shown in Figure 6(b). As might be expected, the spectra become almost indistinguishable from those of PBLG at higher temperatures (above $100^{\circ} \mathrm{C}$ ). This indicates that in the temperature range above the melting point of the stacks of benzyl groups, the characteristic intermolecular interaction for the racemic pair (PBLG-PBDG) cannot be detected as long as it is monitored by fluorescence emission.

\section{CONCLUDING REMARKS}

From the analysis of ${ }^{13} \mathrm{C}-\mathrm{NMR}$ and fluorescence emission spectra, new evidences have been obtained regarding the regularly stacked configuration of benzyl groups ${ }^{1,2}$ formed in the racemic PBG film. Such stacks of benzyl groups exhibit an orderdisorder transition at about $90^{\circ} \mathrm{C}$. Above this temperature, the distinction between the racemic (PBLG + PBDG) and enantiomorphic (PBLG) forms becomes obscure. Similar observations have been reported in the $\operatorname{Raman}^{10}$ and infrared ${ }^{4}$ studies.

Acknowledgment. The authors are grateful to Professor Masahide Yamamoto of Kyoto University for his kind interest in this work.

\section{REFERENCES}

1. Y. Mitsui, Y. Iitaka, and M. Tsuboi, J. Mol. Biol., 24, 15 (1967).

2. J. M. Squire and A. Elliott, J. Mol. Biol., 65, 291 (1972).

3. T. Fukuzawa and I. Uematsu, Polym. J., 6, 537 (1974).

4. M. Yoshikawa, Y. Tsujita, I. Uematsu, and Y. Uematsu, Polym. J., 7, 96 (1975).

5. T. Takahashi, A. Tsutsumi, K. Hikichi, and M. Kaneko, Macromolecules, 7, 806 (1974).

6. J. A. E. Kail, J. A. Sauer, and A. E. Woodward, $J$. Phys. Chem., 66, 1292 (1962).

7. K. Hikichi, J. Phys. Soc. Jpn., 19, 2169 (1964).

8. A. Tsutsumi, S. Anzai, and K. Hikichi, Rep. Prog. Polym. Phys. Jpn., 22, 565 (1979).

9. J. W. Longworth, Biopolymers, 4, 1131 (1966).

10. W. T. Wilser and D. B. Fitchen, Biopolymers, 13, 1435 (1974) 